\title{
The green synthesis and characterization of carboxymethyl cellulose medical dressing with silver nanoparticles
}

\author{
Shan-Fu Zhu ${ }^{1, a}$, Zi-Chao Li ${ }^{2, *}$ \\ Xi-Hui Zhao ${ }^{1,2}$, Yun Xue ${ }^{1,2}$, Jiao-Ying Liu' ${ }^{1}$, Qun $\mathrm{Li}^{1,2, \mathrm{~b}^{*}}$
}

${ }^{1}$ Department of Chemistry Science and Engineering,Qingdao University, Qingdao,266071, China

${ }^{2}$ Collaborative Innovation Center for Marine Biomass Fibers, Materials and Textiles of Shandong

Province, Qingdao University, Qingdao 266071, China

E-mail:azhushanfu2013@163.com, bqunli501@163.com

\begin{abstract}
Keywords: Soluble medical dressings,Nano silver,Carboxymethyl cellulose,Green process, Gel
Abstract. Soluble medical dressing can form gel on the wound surface, which can keep the wound wet and promote wound healing. In this work, carboxymethyl cellulose (CMC) gauze embedded with silver nanoparticles (AgNPs) was prepared via in situ reduction of $\mathrm{Ag}^{+}-\mathrm{CMC}$ gauze in the presence of reducing reagent and stabilizing agent. The effect of reaction conditions such as the reductant, the concentration of $\mathrm{AgNO}_{3}$ and ascorbic acid, reaction time and temperature was studied. The prepared gauze was characterized by UV-vis spectroscopy, Scanning electron microscopy (SEM) and X-ray diffraction (XRD). The results indicated that AgNPs could be formed on the surface of the CMC medical dressing and reaction parameters significantly affected the formation rate, size and distribution of the AgNPs. AgNPs dispersed uniformly on the surface of the fibers when the concentration of $\mathrm{AgNO}_{3}$ and ascorbic acid is $0.1 \mathrm{~mol} / \mathrm{L}$ and $0.2 \mathrm{~mol} / \mathrm{L}$, respectively, with reaction time $35 \mathrm{~min}$ at $30{ }^{\circ} \mathrm{C}$.
\end{abstract}

\section{Introduction}

Medical dressing as covering the wound on broken skin wounds can play a barrier to prevent wound infection, British Winter [1] that "moist wound healing theory", that is wound in a wet state to maintain more favorable healing. Due to an increase in chronic ulcerative burns and wounds, the demand for medical dressings will be growing [2-7].

In accordance dressing medical dressing materials can be divided into the traditional type, natural type and synthetic dressings. Traditional dressings including ordinary cotton cloth, bandages and other dressings such low production costs, but poor water absorption, and can cause adhesion with the wound, the wound secondary injury [8-10].

The starting point of this paper is to overcome the shortcomings of non-aqueous medical materials, preparation of soluble nano silver antimicrobial medical accessories with green synthetic methods. Specific approach is to choose ethanol as solvent system, ascorbic acid as a reducing agent in carboxymethyl modified medical gauze nano crystalline silver, so both soluble and antimicrobial dressing achieve double effect. The new antimicrobial dressings can be widely used in the medical field to heal burns and wounds surface and anti-infection.

\section{Experiments}

\section{Reagents and Instruments}

Reagents: sodium borohydride, ascorbic acid, sodium citrate, glucose, silver nitrate, povidone, ammonia, were of analytical grade, absorbent cotton gauze (purchased in Shandong Overseas Chinese licensing Group Health Materials Co., Ltd.).

Instrument: ZNCL-BS magnetic stirrer, PL303 electronic balance, T6 new century UV-VIS Spectrophotometer, FT-IR200 Fourier transform infrared spectrometer, D/MAX RB X-ray diffraction, TM3000 scanning electron microscope. 


\section{Preparation of carboxymethyl medical dressings}

Carboxymethyl medical dressing with cotton gauze as a raw material, adding a certain concentration of sodium hydroxide solution was basified reaction, after adding a certain amount of acid etherification reaction, and finally to give carboxymethyl cellulose, i.e., soluble fiber medical dressings. Chemical reaction formula shown in Figure 1:

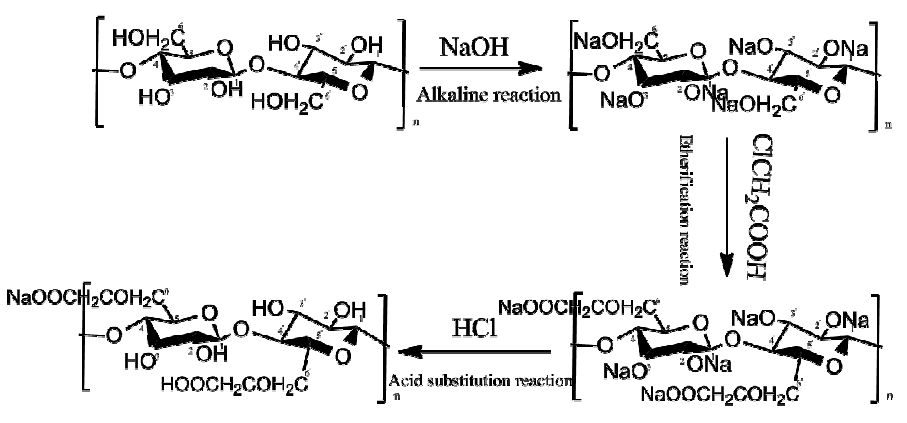

Fig.1. Preparation of soluble fiber medical dressings chemical reaction formula.

\section{Preparation and Characterization of Nano-soluble silver wound dressings}

\section{Preparation}

Carboxymethyl medical dressings soaked in ethanol was added to the system, full swelling, followed by adding different amounts of silver ammonia solution, polyvinylpyrrolidone, stir, ascorbic acid solution was added dropwise, at a certain time at a certain temperature the reaction.

\section{IR}

$\mathrm{KBr}$ tablet method using infrared test for dressing, take $0.5 \mathrm{mg}$ and $2 \mathrm{mg} \mathrm{KBr}$ powder dressing grinding evenly, with FT-IR 200 tests in the wavelength range of $4000 \sim 500 \mathrm{~cm}^{-1}$.

\section{XRD}

With D/MAX-RB X-ray diffraction X-ray diffraction test on the material, $2 \theta$ range of $5^{\circ} \sim 80^{\circ}$, scanning speed $2 \% \mathrm{~min}$.

\section{UV-VIS}

With a UV-VIS spectrophotometer The reaction solution was scanned, scan rate $0.2 \mathrm{~nm} / \mathrm{s}$ in the wavelength range $350 \sim 650 \mathrm{~nm}$.

\section{SEM}

TM3000 scanning electron microscope with the material for spraying pretreatment observation of nano silver and dressing fiber morphology.

\section{Solubility measurement medical dressings}

Respectively shaped clipping ordinary cotton dressing, carboxymethyl medical dressings and medical nano silver carboxymethylcellulose dressing in a petri dish, were added $10 \mathrm{~mL}$ simulated body fluid $(1.42 \mathrm{~mol} / \mathrm{L} \mathrm{NaCl}$ and $0.025 \mathrm{~mol} / \mathrm{L} \mathrm{CaCl} 2)$, dissolved 30min, Comparative morphology changes observed. 


\section{Results and Discussion}

\section{Comparative analysis of infrared spectra}

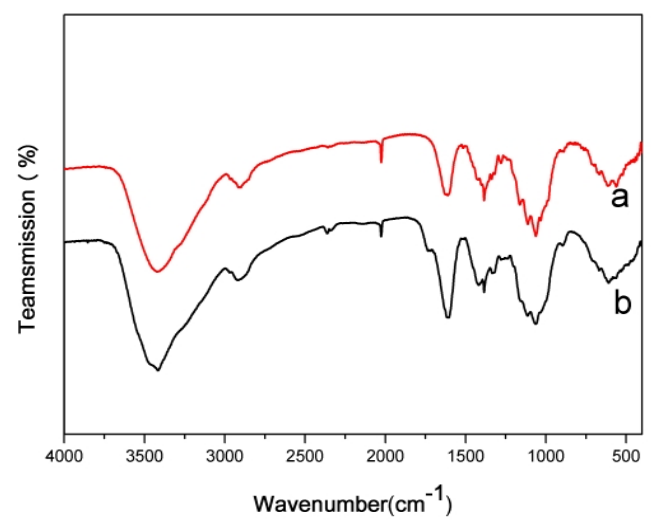

Fig.2. Carboxymethyl medical dressings and medical dressings contained carboxymethylcellulose infrared odd comparison chart.

Fig.2. (b) on behalf of carboxymethyl medical dressings, the absorption peak which $3300 \sim 3400 \mathrm{~cm}^{-1}$ for the hydroxyl group at the stretching vibration peaks in bands around $2855 \sim 2924 \mathrm{~cm}^{-1}$ by an absorption peak at a molecule methyl, methylene antisymmetric and symmetric stretching vibration generated, $1600 \mathrm{~cm}^{-1}$ appeared strong absorption peak belonging carboxyl absorption peak. In 1354 $\sim 1461 \mathrm{~cm}-1$ absorption peak for methyl near the antisymmetric and symmetric bending vibration peak, $1100 \mathrm{~cm}-1$ at the neighborhood, there are $\mathrm{CHO}-\mathrm{CH}_{2}$ bond skeleton vibration peaks within the cellulose ether molecules bond dressing stretching vibration, the deformation vibration absorption peak of hydrogen generated near $650 \sim 750 \mathrm{~cm}^{-1}$.

Fig.2. (a) of nano silver carboxymethylcellulose medical dressings, containing silver infrared spectrum is similar to No silver carboxymethyl medical dressings, indicating that the addition of nano silver change did not cause vibrations of chemical bonds, particularly carboxyl peak at 1600 $\mathrm{cm}^{-1}$ did not change, the silver is deposited on the fiber surface dressing.

\section{UV - visible spectra analysis}
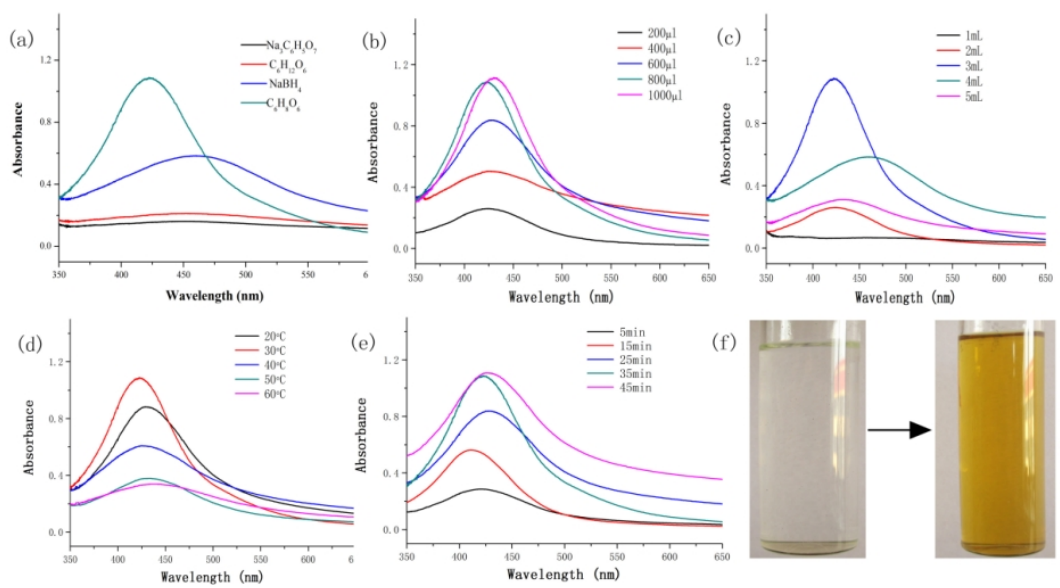

Fig.3. Different conditions UV nano silver reaction liquid visible spectrum.

Depending on the size of nano silver particles, size distribution and concentration of nano-particles with half-width and peak intensity of a great relationship, the position of the absorption peak which can determine the size of the nanoparticles, when the blue-shifted absorption peak position, particle 
size reduction ; conversely, when the absorption peak position redshift, particle size increases. Increase the intensity of the absorption peak, indicating that the amount of silver nano generate more. Fig.3. (a) reducing agent in a different nano-silver, because the sodium citrate insoluble in alcohol, glucose and reducing weak, so there is no generation of nano silver in ethanol systems; and sodium borohydride reduction of the strongest, the reaction will be instantaneous, resulting in the increase of silver nanoparticles aggregate, so this study preferred the reduction of moderate reducing agent ascorbic acid green. Fig.3. (b) is to add different amounts of ascorbic acid reducing agent, with the increase in the amount of ascorbic acid added, nano silver content increases, based on nano silver absorption peak position and intensity, hourly drop added $800 \mu \mathrm{L}$ ascorbic acid, reducing the nano silver best ; Fig.3. (c) is to add various amounts of silver ammonia solution, when added $3 \mathrm{~mL}$, the absorption peak position and intensity for the best, when added to $5 \mathrm{~mL}$, since the $\mathrm{Ag}^{+}$concentration is too large, easy to produce nano silver reunion, will produce larger particles attached to the dressing surface; Fig.3. (d) on behalf of the effects of different reaction temperature on the synthesis of silver nanoparticles, as the temperature rises, the Brownian motion acceleration chance nano silver particle collisions increases, the reaction rate increases, but also produce agglomeration, so at $30{ }^{\circ} \mathrm{C}$, the optimum reaction temperature; Fig.3. (e) the effect of reaction time, as longer reaction time, nano silver increased absorption peak intensity when $35 \mathrm{~min}$ basically unchanged, indicating that the reaction is substantially complete. Fig.3. (f) is the color change of the reaction system, as the reaction proceeded, the system changed from colorless to yellow, indicating that produces nano silver particles.

\section{XRD spectra were compared}

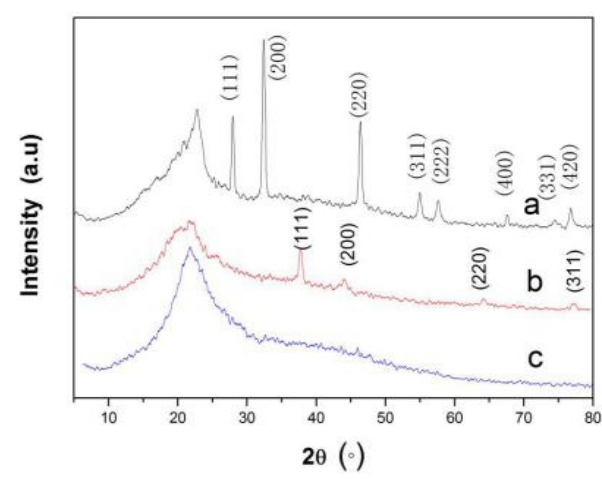

Fig.4. Carboxymethyl medical dressings, surgical dressings containing $\mathrm{AgCl}$ carboxymethyl containing nano-Ag carboxymethyl medical dressings XRD comparison chart

Carboxymethyl medical dressings Fig.4. (a) represents an $\mathrm{AgCl}$, this kind of dressing is due carboxymethylcellulose dressing in the preparation process will be some residual $\mathrm{NaCl}$, if the amount is less than $\mathrm{NH}_{3} \cdot \mathrm{H}_{2} \mathrm{O}, \mathrm{Ag}^{+}$can not be completely envelope together with the $\mathrm{NaCl}$ formed $\mathrm{AgCl}$, so the dressing will $2 \theta 27.83^{\circ}(111), 32.24^{\circ}(200), 46.23^{\circ}(220), 54.83^{\circ}(311), 57.48^{\circ}(222)$, $67.47^{\circ}(400), 74.47^{\circ}(331)$ and $76.73^{\circ}(420) \mathrm{AgCl}$ the XRD characteristic diffraction peaks occurring at. Carboxymethylcellulose medical dressings Fig.4. (b) represents an nanosilver, Fig.4. (c) on behalf of ordinary carboxymethylcellulose medical dressings, visible,(b) at $38.10^{\circ}(111)$, $44.26^{\circ}(200), 64.41^{\circ}(220)$ and $77.46^{\circ}(311)$ appears at the XRD diffraction peaks characteristic of silver nanoparticles, and (c) does not appear, there is only one characteristic diffraction peaks of cellulose, (b)and(c)control Description nano silver has been deposited on Trip Advisor carboxymethylcellulose dressing. 


\section{SEM scanning electron microscopy comparative analysis}

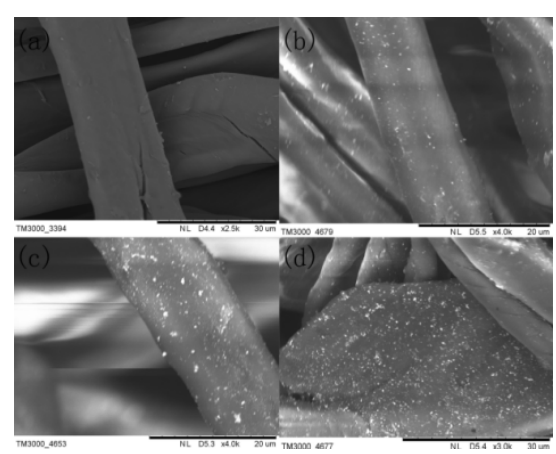

Fig.5. Is deposited under different conditions nanosilver carboxymethylcellulose medical dressings SEM control diagram

Fig.5. (a) is not a load of medical dressings carboxymethyl silver nanoparticles, surface cleaner; Fig.5. (b) is added in an amount of ascorbic acid is too small, nano silver fibers deposited on the less; Fig.5. (c) is a nitric acid silver added excessive agglomeration to produce nano silver particles on the surface of the fiber significantly larger; Fig.5. (d) is a nano silver carboxymethylcellulose medical dressing obtained under optimum reaction conditions, the nano silver particles of moderate size and evenly distributed in the fiber surface.

\section{Dissolved comparison chart analysis}

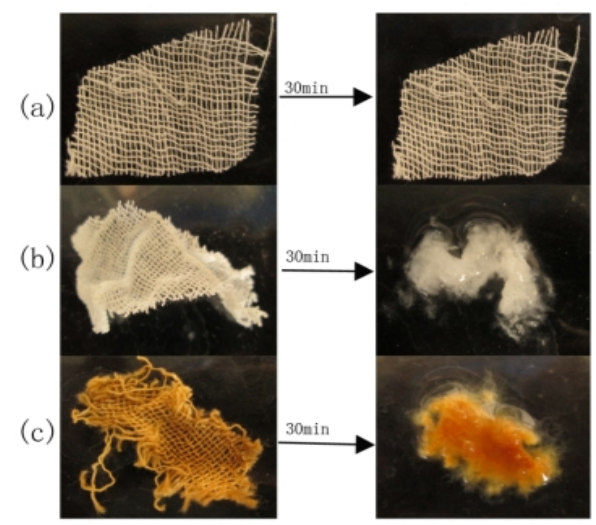

Fig.6. Three kinds of water-soluble medical dressing case comparison chart

Fig.6. (a) on behalf of ordinary cotton dressing dissolved after 30min, the shape did not change in simulated body fluid, described as a water-soluble; (b) on behalf of carboxymethyl cellulose dressings SBF dissolved after 30min, to form water gels, water-soluble better able to keep the wound moist state; (c) on behalf of the nano silver carboxymethyl cellulose dressings SBF dissolved 30min, equally capable of forming a hydrogel, described nano silver added, and It did not change its water solubility characteristics. The incorporation of nano silver antibacterial properties not only increases, but still retains the water into a gel nature, is more excellent performance of new medical accessories.

\section{Conclusion}

Ethanol system, first cotton dressing carboxymethylated, and then restore the silver ammonia ions with ascorbic acid, prepared nano silver carboxymethylcellulose medical dressings. UV-VIS, XRD, SEM prove the fibers are deposited onto the silver nano particles evenly distributed; IR and water soluble nano silver and microscopic proved no chemical bond. Nano silver carboxymethylcellulose 
medical dressings having water into a gel properties.

\section{Acknowledgements}

Postdoctoral Fund,Qing Dao,China.(Project Grant No. 2015134)

\section{Reference}

[1] Kannon GA, Garrett AB. Moist wound healing with occlusive dressings. A clinical review. Dermatol Surg. 1995;21(7):583-590.

[2] Ling Cheng, Yong Gao, Jing Zhou, et al. Classification and characteristics of medical dressings[J]. China Medical Equipment, 2011, 26(5): 99-101.

[3] Rabea EI, Badawy MET, Stevens CV, et al. Chitosan as antimicrobial agent: applications and mode of action $[\mathrm{J}]$. Biomacromolecules, 2003, 4: 1457-1465.

[4] Wedmore I, McManus JG, Pusateri AE, et al. A special report on the chitosan-based hemostatic dressing: Experience in curent combat operations $[\mathrm{J}]$. Journal of Trauma-Injury Infection and Critical Care, 2006, 60( 3) : 655-658.

[5] M IRAFTABM, Qiao Q, Kennedy JF, et al. Fibres for wound dressing s based on mixed carbohydrate po lym er fibres[ J]. Carbohydrate Polymers, 2003, 53( 3) :225-231.

[6] Fan Lihong, Du Yumin , Huang Ronghua , et al .Preparation and characterization of alginate /gelatin blend fibers [J] . Journal of Applied Polymer Science， 2005，96(5):1625-1629.

[7] Yu-Ping Zou,Chun-Lian Zhang,Chen-Bo Cao,et al.Status and Prospects of biological dressing[J].Leather Science and Engineering,2005,15(5):31-35.

[8] Kun-Yu Wang,Zhi-Juan Pan,Jia-Li Chen,et al.Collagen used in medical wound dressing[J].Leather Science and Engineering,2002,12(1):46-49.

[9] Zu-Kun Lu, Li Li, Li-Kai Chu,et al. PVA/PAAS/AM.Preparation and Properties of hydrogel wound dressing[J]. Radiation Research and Radiation Processing, 2013, (3).

[10] Mi F L, Wu Y B, Shyu S S, et al. Asymmetric chitosan membranes prepared by dry/wet phase separation: a new type of wound dressing for controlled antibacterial release[J]. Journal of Membrane Science, 2003, 212(1): 237-254. 